\title{
Pioglitazone is an effective treatment for patients with post-stroke depression combined with type 2 diabetes mellitus
}

\author{
YAOZHI HU ${ }^{1 *}$, HAIYAN XING ${ }^{2 *}$, XIAOMENG DONG ${ }^{1}$, WENXIAN LU ${ }^{1}$, \\ XINXING XIAO $^{1}$, LILIN GAO $^{1}$, MINGHU CUI ${ }^{3}$ and JINBO CHEN ${ }^{1}$ \\ ${ }^{1}$ Department of Neurology, Binzhou Medical University Affiliated Hospital; ${ }^{2}$ Department of Intensive Care Unit, \\ Binzhou People's Hospital; ${ }^{3}$ Department of Psychiatry, Binzhou Medical University Affiliated Hospital, \\ Binzhou, Shandong 256603, P.R. China
}

Received June 4, 2014; Accepted February 6, 2015

DOI: $10.3892 /$ etm.2015.2593

\begin{abstract}
The antidepressive effects of the antidiabetic medicine, pioglitazone, were recently reported in several studies. These effects may ameliorate the depressive symptoms of patients with post-stroke depression (PSD). The present study aimed to evaluate the antidepressive effect of pioglitazone in patients with PSD combined with type 2 diabetes. A total of 118 consecutive patients with stroke who had depression were studied for an average of 3 months. The Diagnostic and Statistical Manual of Mental Disorders (fourth edition) was used to assess whether a patient was depressed or not. The severity of depression was evaluated by the Hamilton depression rating scale (HAMD). In accordance with their HAMD scores, the 118 patients were divided into a severe depression group $(n=40)$ and a mild and moderate $(M M)$ depression group $(\mathrm{n}=78)$. These subjects were then divided into pioglitazone [30 $\mathrm{mg}$ once daily (qd)] and metformin ( $0.5 \mathrm{~g}$ twice daily) subgroups. All patients were given fluoxetine $(20 \mathrm{mg}$ qd). Follow-up evaluations, which included HAMD scores, activities of daily living (ADL) scores, fasting blood glucose (FBG) levels and fasting insulin (FINS) levels, were conducted on the first and third month following the beginning of the treatment. In the MM depression group, the HAMD score in the pioglitazone subgroup was lower than that in the metformin subgroup following treatment for 1 or 3 months. In the severe depression group, the HAMD score in the pioglitazone subgroup was lower than that in the metformin subgroup following 3 months of treatment. The FINS levels of the pioglitazone subgroup gradually decreased in the 3 months
\end{abstract}

Correspondence to: Professor Jinbo Chen, Department of Neurology, Binzhou Medical University Affiliated Hospital, 522 Huanghe 3rd Road, Binzhou, Shandong 256603, P.R. China

E-mail: 707697592@qq.com

*Contributed equally

Key words: post-stroke depression, antidepressant, pioglitazone, type 2 diabetes mellitus of treatment. No noticeable improvement was observed in the ADL scores and FBG values. In conclusion, the results of the current study demonstrate that pioglitazone effectively decreased HAMD scores and FINS values in patients with PSD, suggesting that pioglitazone may be useful for the treatment of patients with PSD combined with type 2 diabetes.

\section{Introduction}

Post-stroke depression (PSD) is one of the most frequent neuropsychiatric diseases following a stroke (1). PSD occurs subsequent to the obvious symptoms of stroke. Patients diagnosed with PSD require the following two conditions: Evident clinical symptoms of stroke, and symptoms of depression following stroke (a patient must meet the diagnostic criteria for depression). The incidence of PSD is $\sim 33 \%$ in all post-stroke patients (2). PSD has serious negative effects on individuals, family and society so its treatment is of great importance.

Pioglitazone, an agonist of peroxisome proliferator-activated receptor (PPAR) $\gamma$, is an antidiabetic drug of the thiazolidinedione class. PPARs mainly exist in insulin-targeted tissues, such as liver, fat and muscle tissues. They ameliorate blood glucose by reducing insulin resistance (3). A number of studies have reported that pioglitazone is effective in the treatment of depression (4-6); however, whether pioglitazone can ameliorate depressive symptoms in patients with PSD remains unknown. In the present study, pioglitazone was administered to patients with PSD and type 2 diabetes mellitus to examine whether pioglitazone can ameliorate the depressive symptoms in such patients.

\section{Materials and methods}

Ethics and consent. This study was approved by the Medical Ethics Committee of Binzhou Medical University Affiliated Hospital (Binzhou, China), and performed in accordance with the Declaration of Helsinki. Enrolled patients provided informed consent.

Subjects. Between April 2012 and January 2014, consecutive patients with stroke who attended the stroke ward of Binzhou Medical University Affiliated Hospital were interviewed to 
assess PSD 3 months after the stroke. Patients were selected for inclusion in the present prospective, randomized controlled study if: i) They were aged $\geq 18$ years; ii) brain magnetic resonance imaging + diffusion-weighted imaging revealed fresh infarctions following admission to the stroke ward; iii) fasting blood glucose (FBG) levels were $\geq 7.0 \mathrm{mmol} / 1$ twice following admission; iv) $6.5<$ glycated hemoglobin (HbA1c) $\leq 11 \%$; v) the body mass index was $\geq 27 \mathrm{~kg} / \mathrm{m}^{2}$; vi) the diagnosis of PSD ( 3 months subsequent to admission) fulfilled the criteria defined in the Diagnostic and Statistical Manual of Mental Disorders (DSM-IV) (7); and vii) the activities of daily living (ADL) score was $>50$ points. Patients were excluded if i) the FBG level was $<7.0 \mathrm{mmol} / 1$ following their stroke; ii) they had a history of vascular disease during the 5 years prior to the stroke; iii) their mini-mental state examination (MMSE) score was $<24$ points; iv) they had severe aphasia following their stroke; v) there was a history of psychiatric disease; vi) they had a medication history of the thiazolidinedione class; and vii) they received administration of other antidiabetic drugs but no insulin injections during the 3 months post-stroke.

Hypertension was diagnosed according to the following values: Systolic blood pressure $\geq 140 \mathrm{mmHg}$ and/or diastolic blood pressure $\geq 90 \mathrm{mmHg}$. Patients who took antihypertensive medications to control blood pressure were classed as being diagnosed with hypertension.

Clinical assessment and outcomes. The experimental group was given fluoxetine [20 mg once daily (qd)] and pioglitazone (30 $\mathrm{mg} \mathrm{qd}$ ) whilst the control group was given fluoxetine (20 mg qd) and metformin (0.5 g twice daily). The two groups were further divided into a mild and moderate (MM) depression and a severe depression group.

Baseline data collected from the patients included age, gender, family status, risk factors, MMSE score and National Institutes of Health stroke scale (NIHSS) score. The following stroke risk factors were identified: i) Hypertension; ii) total cholesterol (TC); iii) high triglyceride levels (TG); iv) high low density lipoprotein (LDL) levels; v) high FBG levels; vi) high fasting insulin (FINS) levels; and vii) high HbAlc levels (8).

The patients were interviewed in order to evaluate their Hamilton depression scale (HAMD) and ADL scores. HAMD scores were assessed and administered by psychiatrists. Neurological physicians were responsible for the ADL, NIHSS and MMSE scores. The follow-up interview was conducted at an average of 3 months following the onset of stroke. The diagnosis of PSD was assessed according to DSM-IV (7). The HAMD scale was also used to simultaneously evaluate MM depression (23 points $\geq$ HAMD score $\geq 8$ points) and severe depression (HAMD score $\geq 24$ points) (9). ADL scores were also assessed at the onset of this interview. The severity of depression was evaluated by HAMD scores following 1 and 3 months of therapy. During the 3 months of therapy, the ADL scores and the levels of FBG and FINS were detected when the treatment was first initiated and at 1 and 3 month time-points.

Statistical analysis. The baseline assessment scores and demographic characteristics were analyzed by appropriate test procedures, including the independent sample t-test and the $\chi^{2}$ test. One-way analysis of variance was used to compare

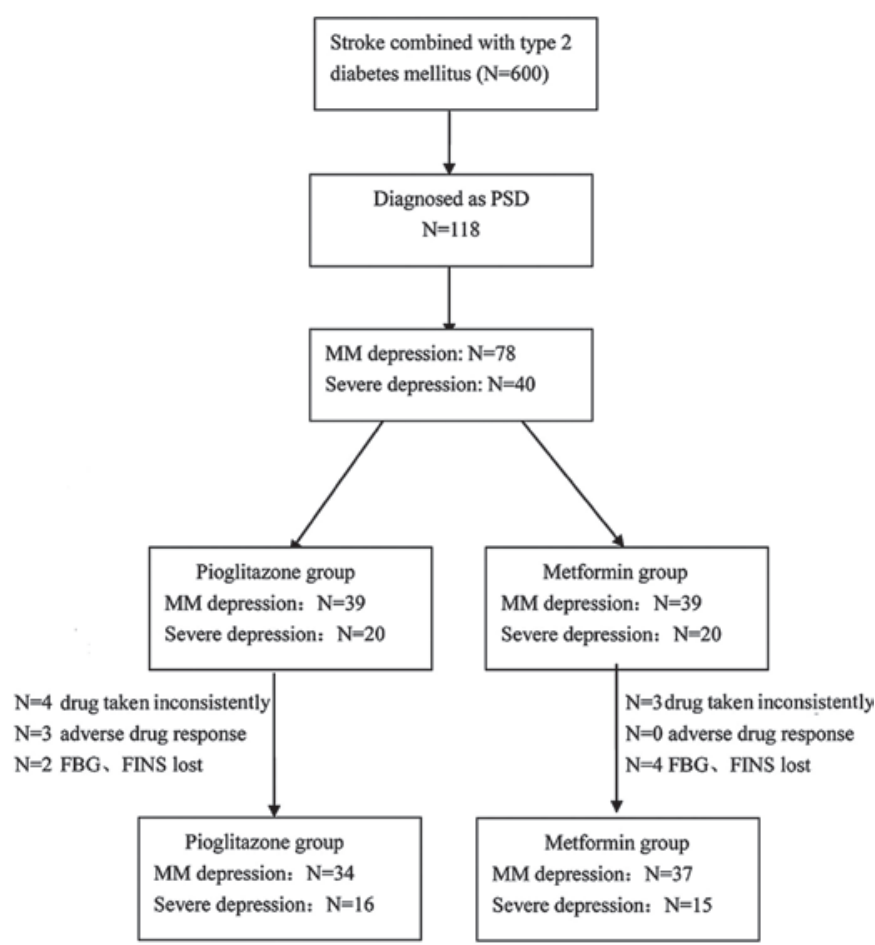

Figure 1. Flow chart showing the selection process of patients with PSD and type 2 diabetes mellitus. PSD, post-stroke depression; MM, mild and moderate depression; FBG, fasting blood glucose; FINS, fasting insulin.

differences between the MM depression and the severe depression groups. Graphpad prism software (version 5.0; Graphpad Software Inc., La Jolla, CA, USA) was used for all statistical analyses. $\mathrm{P}<0.05$ was considered to indicate a statistically significant difference.

\section{Results}

General and baseline characteristics. A total of 600 patients who had acute stroke combined with type 2 diabetes were enrolled in the present study. Of these 600 patients, 470 were excluded since they did not match the DSM-IV criteria and 12 had received antidiabetic drugs other than injected insulin during the 3 months following stroke onset. The remaining 118 patients were diagnosed with PSD according to DSM-IV. The MM depression group comprised 78 patients and the severe depression group comprised 40 patients. These patients were separately distributed into the pioglitazone and metformin subgroups. Each subgroup comprised 39 patients with MM depression and 20 patients with severe depression; however, in the pioglitazone subgroup, 4 patients with severe depression did not consistently take the drugs, and 3 patients with MM depression had a mild adverse drug response during the first month of therapy. Furthermore, the FBG and FINS levels of 2 patients with MM depression were not measured at the correct time during the 3-month therapy. In the metformin subgroup, 3 patients with severe depression did not consistently take the drugs during the first month of therapy, and the FBG and FINS data were lost for 2 patients with MM depression and 2 patients with severe depression after 1 month of therapy (Fig. 1). 
Table I. Sociodemographic and baseline scores of the pioglitazone and metformin groups on admission to the program.

\begin{tabular}{|c|c|c|c|}
\hline Variable & $\begin{array}{l}\text { Pioglitazone group } \\
\qquad(\mathrm{n}=59)\end{array}$ & $\begin{array}{l}\text { Metformin group } \\
\qquad(\mathrm{n}=59)\end{array}$ & P-value \\
\hline Age $^{\mathrm{a}}$ (years) & $63.85 \pm 6.58$ & $65.36 \pm 7.89$ & 0.2619 \\
\hline Gender (female \%) & 61 & 52.5 & 0.3528 \\
\hline Family status & & & 0.6591 \\
\hline Single & 2 & 4 & \\
\hline Married & 25 & 26 & \\
\hline Divorced/widowed & 32 & 29 & \\
\hline Hypertension (n) & 33 & 37 & 0.5742 \\
\hline $\mathrm{TC}^{\mathrm{a}}(\mathrm{mmol} / \mathrm{l})$ & $6.26 \pm 1.60$ & $6.02 \pm 1.90$ & 0.4613 \\
\hline $\mathrm{TG}^{\mathrm{a}}(\mathrm{mmol} / \mathrm{l})$ & $2.73 \pm 1.27$ & $2.91 \pm 1.64$ & 0.5075 \\
\hline $\mathrm{LDL}^{\mathrm{a}}(\mathrm{mmol} / \mathrm{l})$ & $3.92 \pm 1.19$ & $3.60 \pm 1.30$ & 0.1658 \\
\hline $\mathrm{FBG}^{\mathrm{a}}$ & $9.27 \pm 1.71$ & $8.85 \pm 1.72$ & 0.1764 \\
\hline FINS $^{a}$ & $12.20 \pm 2.69$ & $11.43 \pm 2.85$ & 0.1313 \\
\hline $\mathrm{HbA}_{1 c^{\mathrm{a}}}(\%)$ & $8.89 \pm 1.52$ & $8.61 \pm 1.67$ & 0.2676 \\
\hline
\end{tabular}

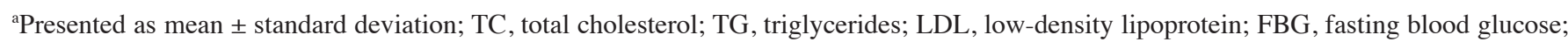
FINS, fasting insulin; HbA1c, glycated hemoglobin.

Table II. ADL and HAMD scores of the pioglitazone and metformin groups during 3 months of therapy.

\begin{tabular}{|c|c|c|c|c|c|c|}
\hline \multirow{2}{*}{$\begin{array}{l}\text { Neuropsyciatric } \\
\text { scores }\end{array}$} & \multicolumn{2}{|c|}{ Pioglitazone group $(n=59)$} & \multicolumn{2}{|c|}{ Metformin group $(n=59)$} & \multirow[b]{2}{*}{ F-value } & \multirow[b]{2}{*}{ P-value } \\
\hline & $\mathrm{MMD}^{\mathrm{a}}$ & Severe $\mathrm{D}^{\mathrm{b}}$ & $\mathrm{MMD}^{\mathrm{c}}$ & Severe $\mathrm{D}^{\mathrm{d}}$ & & \\
\hline \multicolumn{7}{|l|}{ At 0 months } \\
\hline NIHSS & $3.54 \pm 2.33$ & $6.15 \pm 2.70$ & $3.56 \pm 2.40$ & $5.75 \pm 2.69$ & 8.328 & $<0.0001$ \\
\hline MMSE & $26.31 \pm 1.45$ & $26.55 \pm 1.54$ & $26.79 \pm 1.64$ & $26.45 \pm 1.43$ & 0.6839 & 0.5636 \\
\hline ADL & $74.10 \pm 9.38$ & $62.75 \pm 8.35$ & $72.44 \pm 7.68$ & $60.75 \pm 7.83$ & 16.95 & $<0.0001$ \\
\hline HAMD & $21.13 \pm 3.64$ & $44.35 \pm 6.11$ & $20.46 \pm 4.06$ & $46.45 \pm 8.05$ & 39.46 & $<0.0001$ \\
\hline \multicolumn{7}{|l|}{ At 1 month } \\
\hline ADL & $75.14 \pm 9.22$ & $63.75 \pm 9.04$ & $73.72 \pm 8.41$ & $60.59 \pm 7.26$ & 16.04 & $<0.0001$ \\
\hline HAMD & $17.86 \pm 4.05$ & $41.63 \pm 6.10$ & $19.74 \pm 3.35$ & $43.59 \pm 7.67$ & 112.6 & $<0.0001$ \\
\hline \multicolumn{7}{|l|}{ At 3 months } \\
\hline ADL & $77.94 \pm 8.35$ & $64.06 \pm 9.87$ & $75.14 \pm 10.31$ & $63.00 \pm 7.97$ & 14.47 & $<0.0001$ \\
\hline HAMD & $16.59 \pm 3.58$ & $35.25 \pm 5.69$ & $19.32 \pm 3.49$ & $40.60 \pm 8.41$ & 115.6 & $<0.0001$ \\
\hline
\end{tabular}

Scores are presented as mean \pm standard deviation. ${ }^{a} \mathrm{n}=39$ at 0 months, 36 at 1 month and 34 at 3 months; ${ }^{\mathrm{b}}=20$ at 0 months and 16 at 1 and 3 months; ${ }^{c} n=39$ at 0 and 1 months and 37 at 3 months; dn=20 at 0 months, 17 at 1 month and 15 at 3 months. NIHSS, National Institutes of Health stroke scale; MMSE, mini mental state exam; ADL, activities of daily living; HAMD, Hamilton depression scale; MMD, mild and moderate depression; Severe D, severe depression.

No significant differences were observed between the pioglitazone and metformin subgroups regarding sociodemographic and baseline variables. Nevertheless, the severe depression group had higher NIHSS values than the MM depression group $(\mathrm{F}=8.328, \mathrm{P}<0.0001$; Tables I and II).

At 1 month. In the MM depression group, the pioglitazone subgroup $(\mathrm{n}=36)$ had lower HAMD scores compared with the metformin subgroup $(\mathrm{n}=39)$ following 1 month of therapy $(\mathrm{P}=0.0302)$; however, no significant difference was observed in HAMD scores between the metformin subgroup $(n=17)$ and the pioglitazone subgroup $(n=16)$ in the severe depression group. The FINS levels of the pioglitazone group at 1 month $(n=52)$ were lower than those at onset $(n=59 ; P=0.0103)$; however, no such change in FINS levels was detected in the metformin group following 1 month of therapy. The 


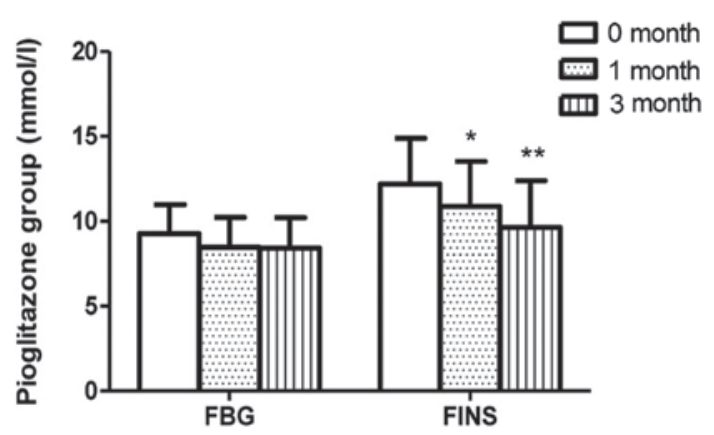

Figure 2. FBG and FINS levels in the pioglitazone subgroup. No changes in FBG levels were found during 3 months of therapy in the pioglitazone subgroup. FINS levels detected following 1 month of therapy $(n=52)$ were lower than those at the onset $\left(\mathrm{n}=59 ;{ }^{*} \mathrm{P}=0.0103\right)$. FINS levels detected after 3 months of therapy $(n=50)$ were lower than those detected after 1 month of therapy $\left(\mathrm{n}=52 ;{ }^{* *} \mathrm{P}=0.0246\right)$. Data are presented as mean \pm standard deviation. FBG, fasting blood glucose; FINS, fasting insulin.

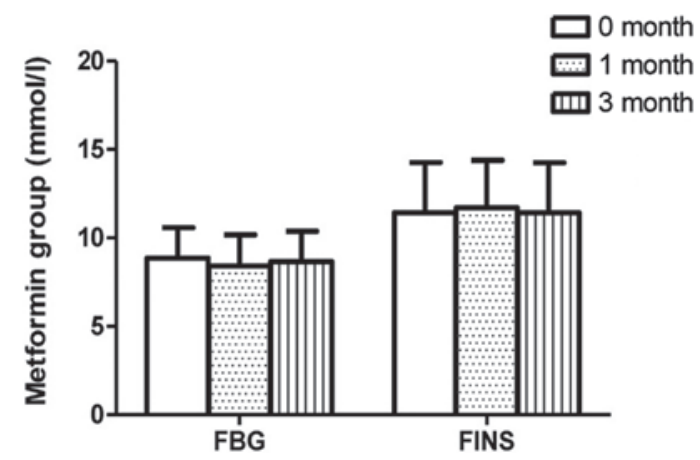

Figure 3. FBG and FINS levels in the metformin subgroup. No changes were found in the levels of FBG and FINS during 3 months of therapy. Data are presented as mean \pm standard deviation. FBG, fasting blood glucose; FINS, fasting insulin.

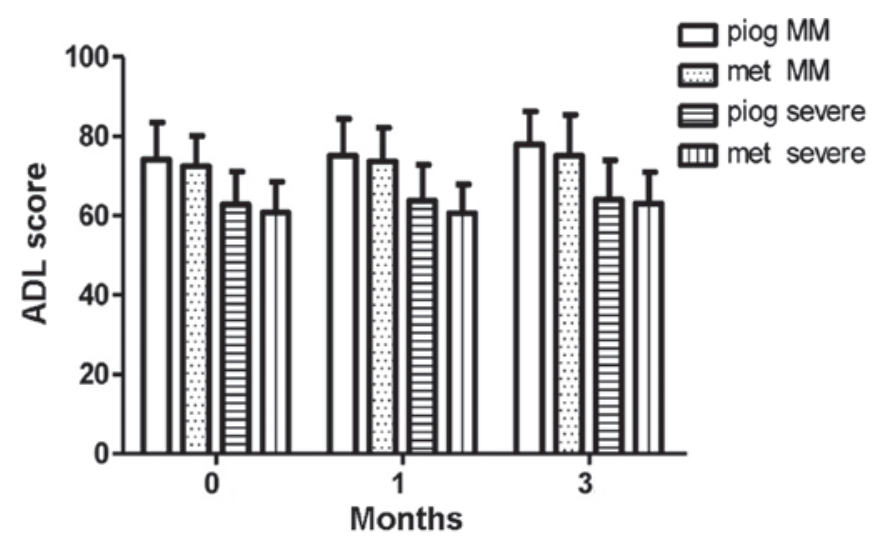

Figure 4. ADL scores during the 3-month therapy period. The ADL score in the MM depression group was higher than that in the severe depression group; however, no difference was found between the pioglitazone and metformin subgroups of the MM depression and severe depression groups. Data are presented as mean \pm standard deviation. ADL, activities of daily living; MM, mild to moderate depression;piog, pioglitazone; met, metformin.

FBG levels were observed to be stable in the pioglitazone and metformin groups (Figs. 2 and 3). No differences in ADL scores were observed between the pioglitazone and metformin groups, in either the MM depression or severe depression subgroups (Fig. 4).

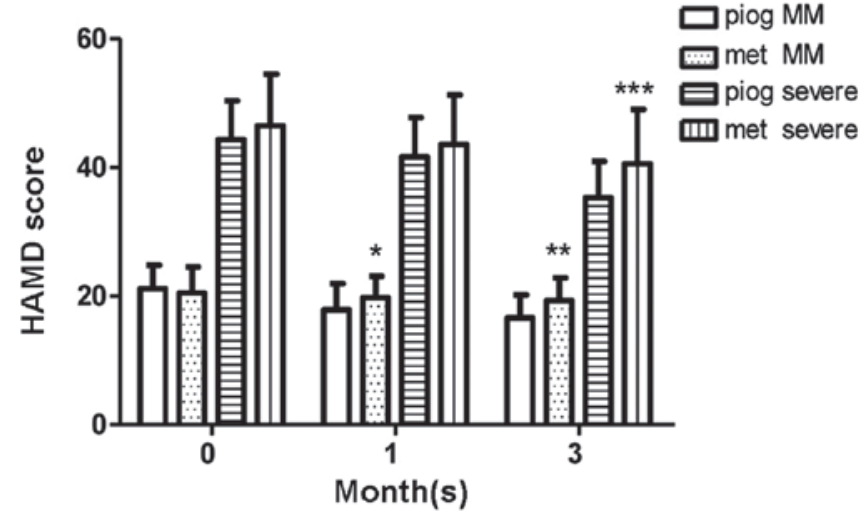

Figure 5. HAMD scores during the 3-month therapy period. In the MM depression group, the pioglitazone subgroup $(n=36)$ had lower HAMD scores than the metformin subgroup $(n=39)$ following 1 month of therapy $\left({ }^{*} \mathrm{P}=0.0302\right)$. In the $\mathrm{MM}$ depression $\left({ }^{* *} \mathrm{P}=0.0017\right)$ and severe depression groups $\left({ }^{* * *} \mathrm{P}=0.0457\right)$, the pioglitazone subgroups ( $\mathrm{MM}$ depression, $\mathrm{n}=34$; severe depression, $\mathrm{n}=16$ ) presented lower HAMD scores than the metformin subgroups (MM depression, $n=37$; severe depression, $n=15$ ) after 3 months of therapy. Data are presented as mean \pm standard deviation. MM, mild to moderate depression; HAMD, Hamilton depression scale; piog, pioglitazone; met, metformin.

At 3 months. In the severe depression group, HAMD scores were lower in the pioglitazone subgroup $(n=16)$ than those in the metformin group $(n=15)$ after 3 months of therapy $(\mathrm{P}=0.0457)$. A similar result was observed in the MM depression group $(\mathrm{P}=0.0017)$. In the pioglitazone group, the FINS levels detected following 3 months of therapy $(n=50)$ were lower than those detected following 1 month of therapy $(n=52$; $\mathrm{P}=0.0246$ ); however, no such change was exhibited in the metformin group. No difference in FBG level was observed in patients between 1 month of therapy and 3 months of therapy in the pioglitazone and metformin subgroups. ADL scores were also higher in the MM depression group compared with those in the severe depression group $(\mathrm{F}=14.47, \mathrm{P}<0.0001$, Table II). In the MM depression and severe depression groups, ADL scores showed no significant difference between the pioglitazone group and the metformin group after the 3 months therapy (Figs. 2-5).

\section{Discussion}

The present study was, to the best of our knowledge, the first to examine the efficacy of pioglitazone using the HAMD scores of patients with PSD combined with type 2 diabetes mellitus. The antidepressive effects of pioglitazone were observed after 3 months of therapy. Pioglitazone ameliorated the depressive symptoms and decreased FINS levels whereas metformin did not; however, pioglitazone was useful for only MM depression following 1 month of treatment. After 3 months of treatment, pioglitazone was also effective against severe depression. The results suggest that pioglitazone may be useful for the treatment of patients with PSD combined with type 2 diabetes.

In the current study the morbidity of PSD was $19.7 \%$, which did not correspond with a previous study describing the prevalence of PSD as $33.3 \%$ (2). The difference in results may be due to the fact that the patients selected in the present study 
were also diagnosed with type 2 diabetes; numerous inclusion criteria could also support this phenomenon. The present study was initiated 3 months following acute stroke as most studies report that the prevalence of PSD is highest during this period (10) enabling the recruitment of more patients in this period. The prevalence of PSD in female patients was $56.8 \%$ in this study, which is in accordance with a systematic review (11).

Kemp et al (5) reported a patient who had severe depression following 12 weeks of pioglitazone treatment with an initial dose of $15 \mathrm{mg}$ qd and an end-point dose of $30 \mathrm{mg} \mathrm{qd}$. In the study, a marked antidepressant response was demonstrated and a significant improvement in insulin resistance. In addition, Kemp et al (12) reported antidepressive effects and improvements in insulin sensitivity among 23 severe depression patients receiving pioglitazone therapy. Kashani et al (4), however, found that patients with severe depression and polycystic ovarian syndrome who took pioglitazone drugs had lower HAMD scores than patients who took metformin. No difference was found between the onset and end of therapy with respect to insulin resistance. Whether the antidepressive effect of pioglitazone is induced by the amelioration of insulin resistance remains unclear as different studies have reached different conclusions. Numerous studies have reported that pioglitazone can decrease the immobility time in the forced swimming test depression model, which cannot be explained by the amelioration of insulin resistance $(13,14)$. This phenomenon was attributed to N-methyl-D-aspartate receptor signaling. In the present study, FINS levels gradually decreased in the pioglitazone subgroup, which demonstrates that pioglitazone decreased insulin resistance (homeostasis model assessment-estimated insulin resistance was calculated using the formula fasting plasma insulin (IU/1) x fasting plasma glucose (mmol/l)/22.5) (15). Depression could dysregulate the hypothalamic-pituitary-adrenal axis causing the deposition of visceral fat, which could lead to insulin resistance (16). Visceral adipose tissue could also lead to inflammatory cytokine production, further contributing to impaired insulin signaling (17).

Few studies have compared the antidiabetic effects of pioglitazone and metformin. The TODAY Study Group found that pioglitazone combined with metformin is better than metformin alone when used on patients with type 2 diabetes mellitus (18). This combination can stabilize blood glucose levels and improve insulin sensitivity. In the current study, no difference was found in FBG levels between the pioglitazone and metformin subgroups during 3 months of therapy. Patients with severe depression had higher NIHSS scores than patients with MM depression; thus, a patient would more easily succumb to severe depression if they had a more severe stroke. A similar trend was observed in ADL scores, in which the MM depression group had higher ADL scores than the severe depression group. Badaru et al (19) reported that PSD may have a passive effect on functional independence in ADL. In depressed or non-depressed patients the intake of antidepressant drugs within the first month post-stroke would be more likely to improve ADL scores than the intake of antidepressant drugs following the first month post-stroke (20). In this previous review, ADL scores were assessed from 6 months following a stroke to 24 months following a stroke. This observation had minimal association with the present results. No change was observed in ADL scores for the pioglitazone and metformin subgroups during the 3-month treatment period. The mechanism underlying this phenomenon should be explored in future studies.

The present study had several limitations. Firstly, the patients were diagnosed with PSD in accordance with DSM-IV 3 months after stroke; however, several patients suffered from depression between the first to third months following their stroke. This occurrence delayed the optimal therapy time. Secondly, patients with higher MMSE and ADL scores were selected in order for this study to proceed conveniently; thus, the sample population did not represent all patients with PSD. Thirdly, the HbA1c values of the patients were $\leq 11 \%$; certain patients with higher HbAlc were excluded from this study. Finally, patients are recommended to be injected with insulin to control blood glucose levels during the acute stroke period. These patients took pioglitazone or metformin combined with insulin at the start of this project, and the insulin dosage may have varied from one individual to another.

Despite these limitations, the data suggest that pioglitazone can decrease depressive symptoms and improve insulin sensitivity compared with metformin. Although the mechanisms of this phenomenon remain unclear, the administration of pioglitazone to patients with PSD combined with type 2 diabetes mellitus may be considered to decrease their HAMD scores.

\section{Acknowledgements}

This study was partly supported by the Chinese State Natural Science Fund (No. 81301182).

\section{References}

1. Morris PL, Robinson RG and Raphael B: Prevalence and course of depressive disorders in hospitalized stroke patients. Int $\mathrm{J}$ Psychiatry Med 20: 349-364, 1990.

2. Turner A, Hambridge J, White J, et al: Depression screening in stroke: a comparison of alternative measures with the structured diagnostic interview for the diagnostic and statistical manual of mental disorders, fourth edition (major depressive episode) as criterion standard. Stroke 43: 1000-1005, 2012.

3. Smith RC, Jin H, Li C, et al: Effects of pioglitazone on metabolic abnormalities, psychopathology, and cognitive function in schizophrenic patients treated with antipsychotic medication: a randomized double-blind study. Schizophr Res 143: 18-24, 2013.

4. Kashani L, Omidvar T, Farazmand B, et al: Does pioglitazone improve depression through insulin-sensitization? Results of a randomized double-blind metformin-controlled trial in patients with polycystic ovarian syndrome and comorbid depression. Psychoneuroendocrinology 38: 767-776, 2013.

5. Kemp DE, Ismail-Beigi F, Ganocy SJ, et al: Use of insulin sensitizers for the treatment of major depressive disorder: a pilot study of pioglitazone for major depression accompanied by abdominal obesity. J Affect Disord 136: 1164-1173, 2012.

6. Sepanjnia K, Modabbernia A, Ashrafi M, Modabbernia MJ and Akhondzadeh S: Pioglitazone adjunctive therapy for moderate-to-severe major depressive disorder: Randomized double-blind placebo-controlled trial. Neuropsychopharmacology 37: 2093-2100, 2012.

7. Pettersson A, Bengtsson Boström K, Gustavsson P and Ekselius L: Which instruments to support diagnosis of depression have sufficient accuracy? A systematic review. Nord J Psychiatry: March 3, 2015 (Epub ahead of print).

8. Jia J and Chen S (eds): Brain vessel disease. In: Neurology (7th edition). People's Medical Publishing House, Beijing, China, p199, 2013.

9. Zimmerman M, Martinez JH, Young D, Chelminski I and Dalrymple K: Severity classification on the Hamilton Depression Rating Scale. J Affect Disord 150: 384-388, 2013. 
10. Schneider MA and Schneider MD: Recognizing poststroke depression. Nursing 42: 60-63, 2012.

11. Poynter B, Shuman M, Diaz-Granados N, Kapral M, Grace SL and Stewart DE: Sex differences in the prevalence of post-stroke depression: a systematic review. Psychosomatics 50: 563-569, 2009.

12. Kemp DE, Ismail-Beigi $\mathrm{F}$ and Calabrese JR: Antidepressant response associated with pioglitazone: support for an overlapping pathophysiology between major depression and metabolic syndrome. Am J Psychiatry 166: 619, 2009.

13. Salehi-Sadaghiani M, Javadi-Paydar M, Gharedaghi MH, et al: NMDA receptor involvement in antidepressant-like effect of pioglitazone in the forced swimming test in mice. Psychopharmacology (Berl) 223: 345-355, 2012.

14. Sadaghiani MS, Javadi-Paydar M, Gharedaghi MH, Fard YY and Dehpour AR: Antidepressant-like effect of pioglitazone in the forced swimming test in mice: The role of PPAR-gamma receptor and nitric oxide pathway. Behav Brain Res 224: 336-343, 2011.

15. Tamayo T, Jacobs DR Jr, Strassburger K, et al: Race-and sex-specific associations of parental education with insulin resistance in middle-aged participants: the CARDIA study. Eur J Epidemiol 27: 349-355, 2012.
16. Austin AW, Gordon JL, Lavoie KL, Arsenault A, Dasgupta K and Bacon S: Differential association of insulin resistance with cognitive and somatic symptoms of depression. Diabet Med 31: 994-1000, 2014.

17. Hardy OT, Czech MP and Corvera S: What causes the insulin resistance underlying obesity? Curr Opin Endocrinol Diabetes Obes 19: 81-87, 2012.

18. TODAY Study Group: Effects of metformin, metformin plus rosiglitazone, and metformin plus lifestyle on insulin sensitivity and $\beta$-cell function in TODAY. Diabetes Care 36: 1749-1757, 2013.

19. Badaru UM, Ogwumike OO, Adeniyi AF and Olowe OO: Variation in functional independence among stroke survivors having fatigue and depression. Neurol Res Int 2013: 842980, 2013.

20. Robinson RG and Spalletta G: Poststroke depression: a review. Can J Psychiatry 55: 341-349 2010. 